\title{
Transient THz photoconductivity in dynamically screened InGaN/GaN quantum wells
}

\author{
Jin, Z.; Lahmann, S.; Rossow, U.; Hangleiter, A.; Bonn, M.; Turchinovich, Dmitry
}

Published in:

Proceedings of the 38th International Conference on Infrared, Millimeter and Terahertz Waves IRMMW-THz 2013

Link to article, DOI:

10.1109/IRMMW-THz.2013.6665920

Publication date:

2013

Link back to DTU Orbit

Citation (APA):

Jin, Z., Lahmann, S., Rossow, U., Hangleiter, A., Bonn, M., \& Turchinovich, D. (2013). Transient THz photoconductivity in dynamically screened InGaN/GaN quantum wells. In Proceedings of the 38th International Conference on Infrared, Millimeter and Terahertz Waves IRMMW-THz 2013 IEEE. https://doi.org/10.1109/IRMMW-THz.2013.6665920

\section{General rights}

Copyright and moral rights for the publications made accessible in the public portal are retained by the authors and/or other copyright owners and it is a condition of accessing publications that users recognise and abide by the legal requirements associated with these rights.

- Users may download and print one copy of any publication from the public portal for the purpose of private study or research.

- You may not further distribute the material or use it for any profit-making activity or commercial gain

- You may freely distribute the URL identifying the publication in the public portal 


\title{
Transient THz Photoconductivity in Dynamically Screened InGaN/GaN Quantum Wells
}

\author{
Z. Jin ${ }^{\mathrm{a}, \mathrm{b}}$, S. Lahmann ${ }^{\mathrm{c}}$, U. Rossow ${ }^{\mathrm{c}}$, A. Hangleiter ${ }^{\mathrm{c}}$, M. Bonn ${ }^{\mathrm{a}}$, and D. Turchinovich ${ }^{\mathrm{a}, \mathrm{d}}$ \\ ${ }^{a}$ Max Planck Institute for Polymer Research, 55128 Mainz, Germany \\ ${ }^{\mathrm{b}}$ Shanghai University, 200444 Shanghai, China \\ ${ }^{\mathrm{c}}$ Technical University of Braunschweig, 38106 Braunschweig, Germany \\ ${ }^{\mathrm{d}}$ Technical University of Denmark, 2800 Lyngby, Denmark
}

\begin{abstract}
Using optical pump - THz probe spectroscopy we reveal complex ultrafast photoconductivity dynamics in InGaN/GaN quantum wells under dynamical screening conditions, where, at sufficiently high excitation densities, the photo-generated carriers fully screen the initial internal field of 3 $\mathrm{MV} / \mathrm{cm}$. The THz photoconductivity spectra contain features of both localized and free charges.
\end{abstract}

\section{INTRODUCTION AND BACKGROUND}

$\mathrm{Q}$ UANTUM wells (QWs) in the presence of electric field are well described within the quantum-confined Stark effect (QCSE) formalism. The electric field breaks the symmetry, decreasing the optical transition energy and reducing interband matrix element (and hence absorption and recombination rates) via spatial separation of electron and hole wavefunctions. However, strong and ultrafast (i.e. on the timescale much faster than the recombination time) optical excitation of the biased quantum well will lead to a coherent dynamical screening effect (CDS), as a significant photogenerated population of polarized electrons and holes will decrease, or even completely screen the initial bias field in the QWs ${ }^{[1-4]}$. In the latter case, the initial QCSE can be completely removed on timescales shorter than the duration of the excitation pulse, leaving the QW in the screened "rectangular-shape" state. As a result, the QCSE is removed, and the optical transition energy and the recombination rate increase. However, as the carriers recombine, the bias field, and hence the QCSE, gradually restore. The complete process is depicted in Fig. 1.

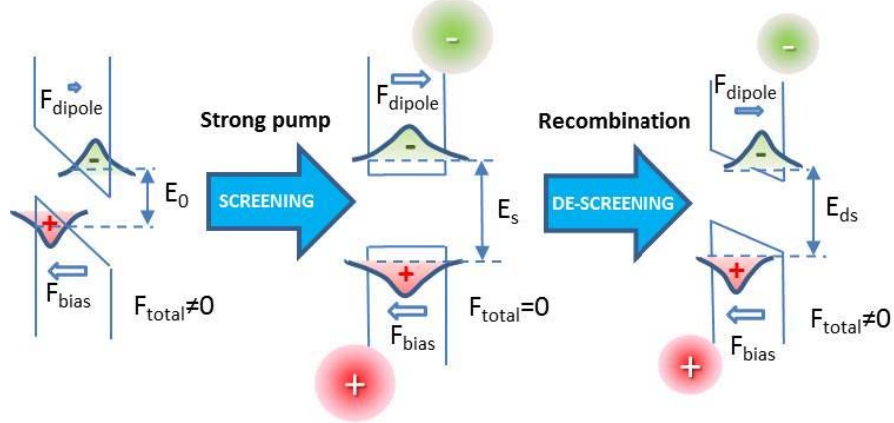

Fig. 1. Principle of dynamical screening effect in a biased QW. Strong optical excitation produces a polarized screening charges (shown as red and green "clouds") of sufficient density to compensate the initial bias field, removing the QCSE. As the screening charges recombine, the bias field and the QCSE are restored.

\section{RESULTS}

Rigorous optical pump-THz probe (OPTP) experiments reveal the photoconductivity dynamics of the screening charge in internally-biased $\mathrm{InGaN} / \mathrm{GaN}$ QWs excited with variable fluence at $400 \mathrm{~nm}$. Our sample consists of 10 periods of $\mathrm{In}_{0.2} \mathrm{Ga}_{0.8} \mathrm{~N}$ QWs with a well thickness of $1.8 \mathrm{~nm}$, separated by $\mathrm{GaN}$ barriers with the width of $7 \mathrm{~nm}$. The strain-induced built-in field in the QWs, caused by QW-barrier lattice mismatch, was $3.1 \mathrm{MV} / \mathrm{cm}^{[1]}$. The multi-QWs structure is sandwiched between a $180 \mathrm{~nm}$ thick GaN capping layer and a 2 $\mu \mathrm{m} \mathrm{GaN} \mathrm{buffer} \mathrm{layer.} \mathrm{The} \mathrm{sample} \mathrm{was} \mathrm{grown} \mathrm{on} \mathrm{a} \mathrm{sapphire}$ substrate by metalorganic vapor-phase epitaxy. The $400 \mathrm{~nm}$ excitation wavelength assures that with single-photon absorption, electron-hole pairs can only be excited in the InGaN QWs and not in the GaN barriers. In order to assess the presence of the QCSE, time-integrated photoluminescence (PL) measurements were performed with different excitation fluences. The photoexcitation-induced screening and subsequent de-screening of the built-in field was monitored using the time-integrated photoluminescence (PL, data not shown). The saturation of the blue-shift of the main PL feature for highest fluences indicates the removal of Stark shift, and thus the complete screening of the built-in piezoelectric field ${ }^{[1,2]}$. As carriers recombine, a decrease of the transition energy results in a red-shift of the PL spectra, as the QCSE returns. Complete screening is achieved already at pump fluences of $\sim 0.5 \mathrm{~mJ} / \mathrm{cm}^{2}$, leading to a non-trivial speed-up of the screening charge decay, as described below.

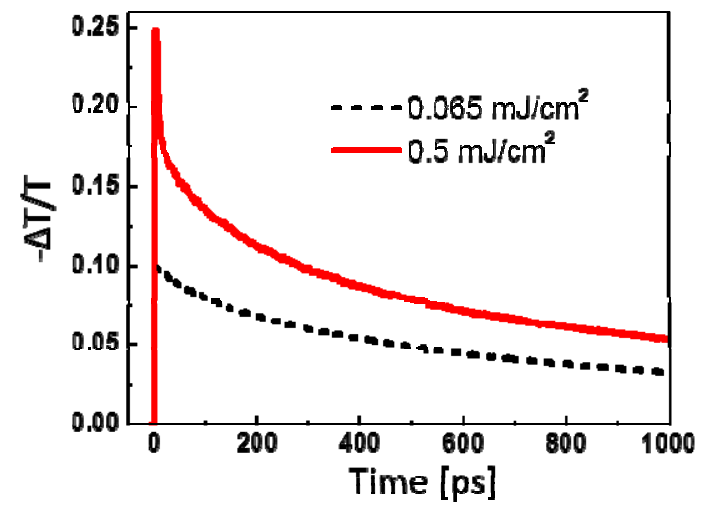

Fig. 2 Frequency-integrated $\mathrm{THz}$ transmission through the QW sample photoexcited by $400 \mathrm{~nm}$, sub-50 fs pulses at weak $(0.065$ $\mathrm{mJ} / \mathrm{cm}^{2}$, insignificant screening) and strong $\left(0.5 \mathrm{~mJ} / \mathrm{cm}^{2}\right.$, complete screening) pump fluence.

Fig. 2 shows the $\mathrm{THz}$ frequency-integrated transient photoconductivity in InGaN/GaN QWs as measured in a 1D OPTP experiment for two pump fluences, $0.065 \mathrm{~mJ} / \mathrm{cm}^{2}$ and 0.5 $\mathrm{mJ} / \mathrm{cm}^{2}$. A non-exponential decay of the photoconductivity is 
clearly evident (with an accelerated initial decay) at larger pump fluences. At high pump fluences and early pump-probe delay times, the complete screening of the built-in piezoelectric field results in a large overlap of the electron and hole wave functions and hence a large recombination rate. At later pump-probe delay times, the carrier density decreases due to electron-hole recombination, resulting in the restoration of the built-in piezoelectric field, which in turn decreases the recombination rate.

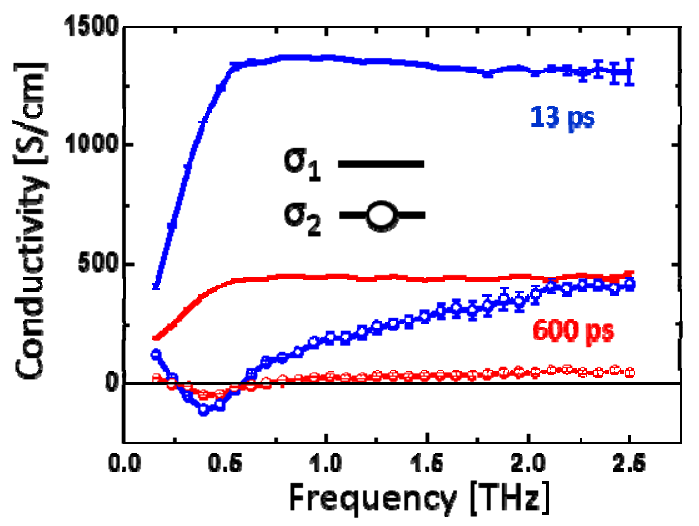

Fig.3 Real $\left(\sigma_{1}\right)$ and imaginary $\left(\sigma_{2}\right) \mathrm{THz}$ conductivity spectra at screening photoexcitation of $0.5 \mathrm{~mJ} / \mathrm{cm}^{2}$, measured $13 \mathrm{ps}$ and $600 \mathrm{ps}$ after pump. The QW thickness is $1.8 \mathrm{~nm}$.

Spectral information about $\mathrm{THz}$ photoconductivity dynamics can be obtained by time-resolving the probe $\mathrm{THz}$ pulses at various times after optical excitation, and performing the spectral analysis in the Fourier domain. The complex-valued $\mathrm{THz}$ frequency-resolved transmission function can be related to the photoinduced complex THz conductivity, $\widetilde{\sigma}(\omega)$, at any time delay after the photoexcitation, given that $\widetilde{\sigma}(\omega)$ varies slowly relative to the duration of the $\mathrm{THz}$ pulse $(\sim 1 \mathrm{ps})$.

Fig. 3 shows such complex conductivity spectra measured at two pump-probe delays. To the best of our knowledge, this is the first spectrally-resolved observation of ultrafast photoconductivity dynamics in a GaN-based system. We have found that the shape of the spectra stays unchanged within the whole range of our pump-probe delays $13-800 \mathrm{ps}$, while only decreasing in magnitude with time. Reduced low-frequency conductivity and the zero-crossing of the imaginary part of the conductivity at about $0.6 \mathrm{THz}$ indicate a non-Drude conductivity response. The measured spectra could not be fitted by any simple analytical conductivity model [Drude (-Smith), Drude-Lorentz], however their features can still lead to qualitative conclusions regarding the nature of photoconductivity in InGaN/GaN QWs system.

At lowest $\mathrm{THz}$ frequencies the impeded $\mathrm{DC}$ real conductivity, its growth with frequency, and negative imaginary conductivity suggest the "polarization-type" response of bound or localized charge. At higher frequencies, spectrally-flat real conductivity and slowly-growing positive imaginary conductivity suggest free carrier conductivity at high momentum relaxation rate. These features are indicative of carrier localization on the long-range corrugated potential energy surface in InGaN/GaN QWs ${ }^{[5]}$, which may have an impact on the performance of GaN-based devices, e.g. laser diodes and lasers in the blue-green spectral region, and is currently the subject of further investigations.

In summary, we have observed a speed-up in the decay of the photoexcited screening charge at increased excitation fluences, showing an increase in recombination (or trapping) rate in the screened QWs. The time-resolved $\mathrm{THz}$ conductivity spectra at various time delays after optical excitation show non-Drude-like behavior, which we tentatively attribute to the localization of free charge on long-range potential fluctuations.

\section{REFERENCES}

[1] D. Turchinovich, P. Uhd Jepsen, B. S. Monozon, M. Koch, S. Lahmann, U. Rossow, and A. Hangleiter, "Ultrafast polarization dynamics in biased quantum wells under strong femtosecond optical excitation" Phys. Rev. B 68, 241307 (R) (2003).

[2] D. Turchinovich, B. S. Monozon, and P. Uhd Jepsen, "Role of dynamical screening in excitation kinetics of biased quantum wells: Nonlinear absorption and ultrabroadband terahertz emission", J. Appl. Phys. 99, 013510 (2006).

[3] P. J. S. van Capel, D. Turchinovich, H. P. Porte, S. Lahmann, U. Rossow, A. Hangleiter, and J. I. Dijkhuis, "Correlated terahertz acoustic and electromagnetic emission in dynamically screened $\mathrm{InGaN} / \mathrm{GaN}$ quantum wells", Phys. Rev. B 84, 085317 (2011).

[4] H. P. Porte, D. Turchinovich, D. G. Cooke and P. Uhd Jepsen, "Terahertz study of ultrafast carrier dynamics in $\mathrm{InGaN} / \mathrm{GaN}$ multiple quantum wells", J. Phys.: Conf. Ser. 193, 012084 (2009).

[5] A. Hangleiter, F. Hitzel, C. Netzel, D. Fuhrmann, U. Rossow, G. Ade, and P. Hinze, "Suppression of Nonradiative Recombination by V-Shaped Pits in GaInN/GaN Quantum Wells Produces a Large Increase in the Light Emission Efficiency", Phys. Rev. Lett. 95, 127402 (2005). 